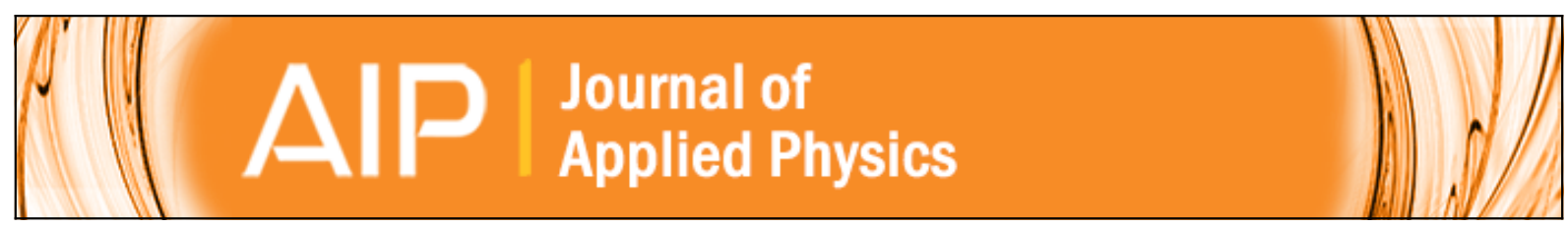

\title{
Spin glass behavior in Sr2Mn0.7Fe0.3MoO6
}

Xianjie Wang, Yu Sui, Yao Li, Miao Xu, Wendong Wang, Pan Liu, and Jinke Tang

Citation: Journal of Applied Physics 109, 07 C322 (2011); doi: 10.1063/1.3562889

View online: http://dx.doi.org/10.1063/1.3562889

View Table of Contents: http://scitation.aip.org/content/aip/journal/jap/109/7?ver=pdfcov

Published by the AIP Publishing

\section{Articles you may be interested in}

Magnetic and transport properties of cobalt doped La0.7Sr0.3MnO3

J. Appl. Phys. 116, 103907 (2014); 10.1063/1.4894713

Observation of spin-glass behavior in antiperovskite compound Mn3Cu0.7Ga0.3N

Appl. Phys. Lett. 103, 022405 (2013); 10.1063/1.4813412

Structural, electrical, magnetic, and thermal studies of Cr-doped La $0.7 \mathrm{Ca} 0.3 \mathrm{Mn} 1-\mathrm{x} \mathrm{Cr} \times \mathrm{O} 3(0 \leq \mathrm{x} \leq 1)$ manganites

J. Appl. Phys. 107, 083905 (2010); 10.1063/1.3342462

Structural and magnetic properties of Co $1-x$ Fe $x$ Sr 2 YCu $207+\delta$ compounds

J. Appl. Phys. 107, 063905 (2010); 10.1063/1.3327452

Evidence of disorder induced magnetic spin glass phase in $\mathrm{Sr} 2 \mathrm{FeMoO} 6$ double perovskite J. Appl. Phys. 106, 073908 (2009); 10.1063/1.3236566






\title{
Spin glass behavior in $\mathrm{Sr}_{2} \mathrm{Mn}_{\mathbf{0 . 7}} \mathrm{Fe}_{0.3} \mathrm{MoO}_{6}$
}

\author{
Xianjie Wang, ${ }^{1,2}$ Yu Sui, ${ }^{1, a)}$ Yao Li, ${ }^{2, b)}$ Miao Xu, ${ }^{1}$ Wendong Wang, ${ }^{3}$ Pan Liu, ${ }^{4}$ \\ and Jinke Tang ${ }^{4}$ \\ ${ }^{1}$ Center for Condensed Matter Science and Technology (CCMST), Department of Physics, \\ Harbin Institute of Technology, Harbin 150001, People's Republic of China \\ ${ }^{2}$ Center for Composite Materials, Harbin Institute of Technology, Harbin 150001, People's Republic of China \\ ${ }^{3}$ Institute of Microelectronics of Chinese Academy of Sciences, Beijing 100029, People's Republic of China \\ ${ }^{4}$ Department of Physics \& Astronomy, University of Wyoming, Laramie, Wyoming 82071, USA
}

(Presented 17 November 2010; received 23 September 2010; accepted 15 December 2010; published online 7 April 2011)

\begin{abstract}
In this paper, we present a detailed study of the dc magnetization, ac susceptibility and aging effect in a polycrystalline $\mathrm{Sr}_{2} \mathrm{Mn}_{0.7} \mathrm{Fe}_{0.3} \mathrm{MoO}_{6}$ sample. Based on the tetragonal I 4/m space group, the XRD pattern is well refined, which shows that the sample is crystallized in a single phase double-perovskite structure without impurity phase. The dc magnetization, ac susceptibility, heat capacity, aging experiments and fitting results show the existence of the spin glass behavior in the sample. The spin glass behavior originates from the competition between the ferromagnetic and antiferromagnetic couplings due to the magnetic frustration at the interface of ferromagnetic and antiferromagnetic clusters in the phase separate sample. (C) 2011 American Institute of Physics. [doi:10.1063/1.3562889]
\end{abstract}

\section{INTRODUCTION}

Recently, much more attention has been paid to the magnetotransport properties of ordered double perovskiten oxides, ${ }^{1-3}$ especially $\mathrm{Sr}_{2} \mathrm{FeMoO}_{6}$, due to their promising potential for spintronic device applications. $\mathrm{Sr}_{2} \mathrm{FeMoO}_{6}$ is a ferromagnetic metal. ${ }^{1}$ The magnetic structure of $\mathrm{Sr}_{2} \mathrm{FeMoO}_{6}$ was generally described as ferrimagnetic due to the antiferromagnetic coupling of localized spin-up electrons of $\mathrm{Fe}^{3+}$ $\left(3 d^{5}: t^{3}{ }_{2 g} e^{2}\right)$ and itinerant spin-down electrons of $\mathrm{Mo}^{5+}$ $\left(4 d^{1}: t^{1}{ }_{2 g}\right)$. Due to the similarity of electronic configurations between $\mathrm{Fe}$ and $\mathrm{Mn}$ ions, a lot of investigations have been done for $\mathrm{Sr}_{2} \mathrm{Fe}_{1-x} \mathrm{Mn}_{x} \mathrm{MoO}_{6}$, especially the magnetoresistance and magnetic properties of Fe-rich samples. ${ }^{4-6}$ When the $\mathrm{Fe}$ ions are replaced by $\mathrm{Mn}$, the Mo ions will lose the itinerant $4 d^{1}$ electron, and the $\mathrm{Sr}_{2} \mathrm{MnMoO}_{6}$ is an antiferromagnetic insulator. ${ }^{7,8}$ Complex magnetic behaviors are observed in the $\mathrm{Sr}_{2} \mathrm{Fe}_{1-x} \mathrm{Mn}_{x} \mathrm{MoO}_{6}$ system.

But less work has been done for Mn-rich samples, which should be more helpful for understanding the evolution of the magnetic properties from the ferromagnetic state to antiferromagnetic state. Recently, stronger magnetic frustration effect was observed in Mn-rich samples by Poddar and Mazumdar. ${ }^{8}$ When the Fe concentration is about $20 \%$, the isolated Fe cluster start to form and the cluster glass phenomenon is observed in $\mathrm{Sr}_{2} \mathrm{Mn}_{1-x} \mathrm{Fe}_{x} \mathrm{MoO}_{6}$. They also suggested that the ferromagnetic order is centered on substituted $\mathrm{Fe}$ atoms. Moritomo et al. also reported a ferromagnetic order for $\mathrm{Fe}$ content as low as $30 \%$ in $\mathrm{Sr}_{2} \mathrm{Mn}_{1-x} \mathrm{Fe}_{x} \mathrm{MoO}_{6}$ samples. ${ }^{6}$ It is well known that the magnetic frustration at the interface of ferromagnetic and antiferromagnetic clusters will result in the spin glass behavior. Poddar et al. further proposed that the spin glass will form due to the cationic disorder in $\mathrm{Sr}_{2} \mathrm{Fe}$ -

\footnotetext{
a) Author to whom correspondence should be addressed. Electronic mail: suiyu@hit.edu.cn.

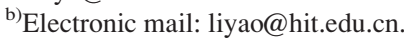

$\mathrm{MoO}_{6}$ (Ref. 9). But the origin of the spin glass remains an open question in $\mathrm{Sr}_{2} \mathrm{Mn}_{1-x} \mathrm{Fe}_{x} \mathrm{MoO}_{6}$ system. It is interesting to explore the magnetic behavior in the complex magnetic system, and less work has been done in $\mathrm{Sr}_{2} \mathrm{Mn}_{1-x} \mathrm{Fe}_{x} \mathrm{MoO}_{6}$. Therefore, detailed investigation about $\mathrm{Sr}_{2} \mathrm{Mn}_{1-x} \mathrm{Fe}_{x} \mathrm{MoO}_{6}$ system is still necessary to understand these magnetic properties more thoroughly.

In this paper, we present a detailed study of the dc magnetization, ac susceptibility and aging effect in a polycrystalline $\mathrm{Sr}_{2} \mathrm{Fe}_{0.3} \mathrm{Mn}_{0.7} \mathrm{MoO}_{6}$ sample. Our results give strong evidence that the spin glass originates from the competition between the ferromagnetic and antiferromagnetic couplings as seen in the Fe-rich and Mn-rich samples, respectively.

\section{EXPERIMENT}

The $\mathrm{Sr}_{2} \mathrm{Mn}_{0.7} \mathrm{Fe}_{0.3} \mathrm{MoO}_{6}$ (SMFMO) polycrystalline samples were prepared by conventional solid-state reaction. The mixture of high purity $\mathrm{SrCO}_{3}, \mathrm{Fe}_{2} \mathrm{O}_{3}, \mathrm{MnO}_{2}$, and $\mathrm{MoO}_{3}$ was pressed into pellets and calcined in air at $1173 \mathrm{~K}$ for $3 \mathrm{~h}$. The pellets were then reground, pressed and reheated with sintering at $1473 \mathrm{~K}$ for $2 \mathrm{~h}$ in a stream of $1.5 \% \mathrm{H}_{2} / \mathrm{N}_{2}$. X-ray diffraction (XRD) powder patterns were collected using a Bede $\mathrm{D}^{1} \mathrm{XRD}$ spectrometer with $\mathrm{Ni}$-filtered $\mathrm{CuK} \alpha$ radiation. The room-temperature XRD patterns were refined by the Rietveld method using the program FULLPROF. ${ }^{10,11}$ The dc magnetization, ac susceptibility and heat capacity measurements were carried out using the physical properties measurement system (PPMS) of Quantum Design.

\section{RESULTS AND DISCUSSION}

Figure 1 shows the XRD patterns of $\mathrm{Sr}_{2} \mathrm{Mn}_{0.7} \mathrm{Fe}_{0.3} \mathrm{MoO}_{6}$ (SMFMO) polycrystalline samples. A good-quality solid solution was achieved and no impurity phase is observed. Based on the tetragonal $I 4 / m$ space group, the XRD pattern is well refined. ${ }^{12}$ The cationic order of $\mathrm{Fe}(\mathrm{Mn})$ and Mo can 


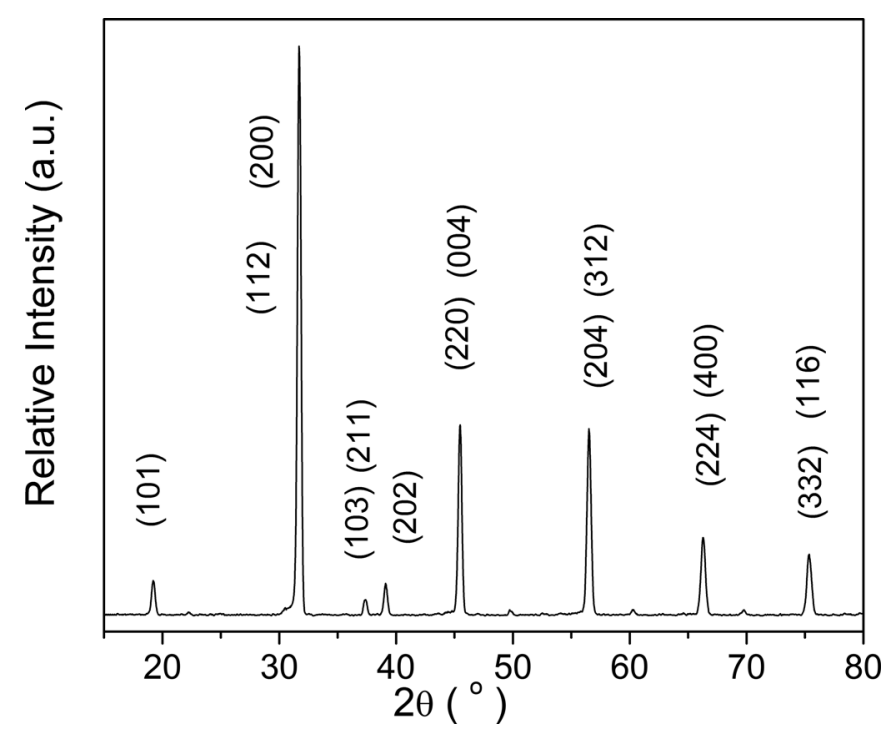

FIG. 1. Room temperature XRD pattern of $\mathrm{Sr}_{2} \mathrm{Mn}_{0.7} \mathrm{Fe}_{0.3} \mathrm{MoO}_{6}$ sample.

be calculated from the refined results, and its value is about $97 \%$, which is consistent with the results of Feng et al. ${ }^{4}$ The stronger diffraction peak of (101) also characterizes the high degree of cationic order and the good-quality of the double perovskite structure. ${ }^{13,14}$

It is well known that the $\mathrm{Sr}_{2} \mathrm{FeMoO}_{6}$ and $\mathrm{Sr}_{2} \mathrm{MnMoO}_{6}$ are ferromagnetic and antiferromagnetic, respectively. And the ferromagnetic and antiferromagnetic clusters will exist in the SMFMO sample. The interaction between the ferromagnetic and antiferromagnetic clusters will lead to a complex magnetic behavior. Figure 2 shows the dc magnetization as a function of temperature measured in a magnetic field of 100 Oe after zero-field-cooled (ZFC) and field-cooled (FC) processes for SMFMO. Both of the ZFC and FC curves show a peak at about $24 \mathrm{~K}$, although the two deviate below the peak. The paramagnetic Curie temperature, calculated from the Curie-Weiss law (1/M vs $T$ curve, see Fig. 2 , inset), is about



FIG. 2. (Color online) Temperature dependence of the dc magnetization after field cooled (closed symbols) and zero field cooled (open symbols). (b) Inset shows the temperature dependence of the $1 / M$.
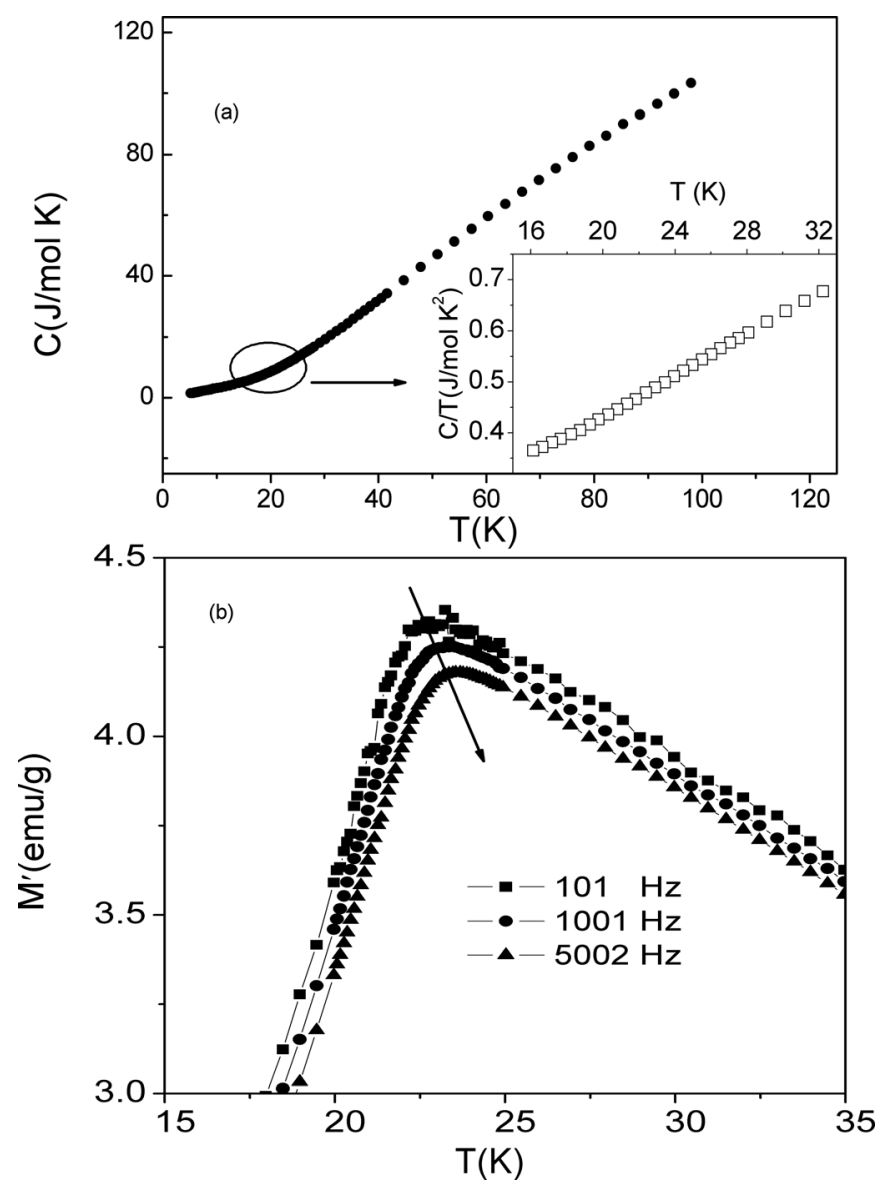

FIG. 3. (a) The heat capacity of $\mathrm{Sr}_{2} \mathrm{Mn}_{0.7} \mathrm{Fe}_{0.3} \mathrm{MoO}_{6}$ sample; (b) the temperature dependence of the ac susceptibility at different frequencies.

$80 \mathrm{~K}$, which implies ferromagnetic coupling. Similar result was also observed by Poddar and Mazumdar. ${ }^{8}$ Figure 3(a) shows the specific heat capacity curve of SMFMO. There is no apparent peak near $24 \mathrm{~K}$ both in the specific capacity and $C / T$ curves, as shown in Fig. 3(a) and its inset. This suggests what is observed at $24 \mathrm{~K}$ is a spin glass or superparamagnetism behavior instead of the antiferromagnetic behavior.

To explore the origin of the magnetic properties, we measure the ac susceptibility of SMFMO at different frequencies. Figure 3(b) shows the ac susceptibility as a function of temperature. The peaks of $M^{\prime}(T)$ show frequency dependence and occur very close to the peak of ZFC curve, which is usually observed at the freezing temperature of a spin glass. As seen, the peak temperature increases with increasing frequency. It is well known that the frequency dependence in spin-glass system can be described as $\tau / \tau_{0}=\left[\left(T-T_{\mathrm{SG}}\right) / T_{\mathrm{SG}}\right]^{-\mathrm{z}}$, where $\tau$ is the measured relaxation time, $T_{\mathrm{SG}}$ is the critical temperature of spin-glass ordering, $\mathrm{z}$ is the dynamical exponent, and $\tau_{0}$ is the characteristic time scale of the spin glass. ${ }^{15}$ This equation fits our experimental data excellently, and the fitting parameters of $T_{\mathrm{SG}}, z$ and $\tau_{0}$ are $20.94 \mathrm{~K}, 4.28$ and $2.70 \times 10^{-12} \mathrm{~s}$, respectively. These results suggest the existence of the spin glass behavior in the sample, which are consistent with the results of dc magnetization and special heat capacity.

To further confirm the nature of the spin glass behavior in SMFMO, we measured the magnetic relaxation and aging curve at $10 \mathrm{~K}$. The sample was cooled from 300 to $10 \mathrm{~K}$ in 

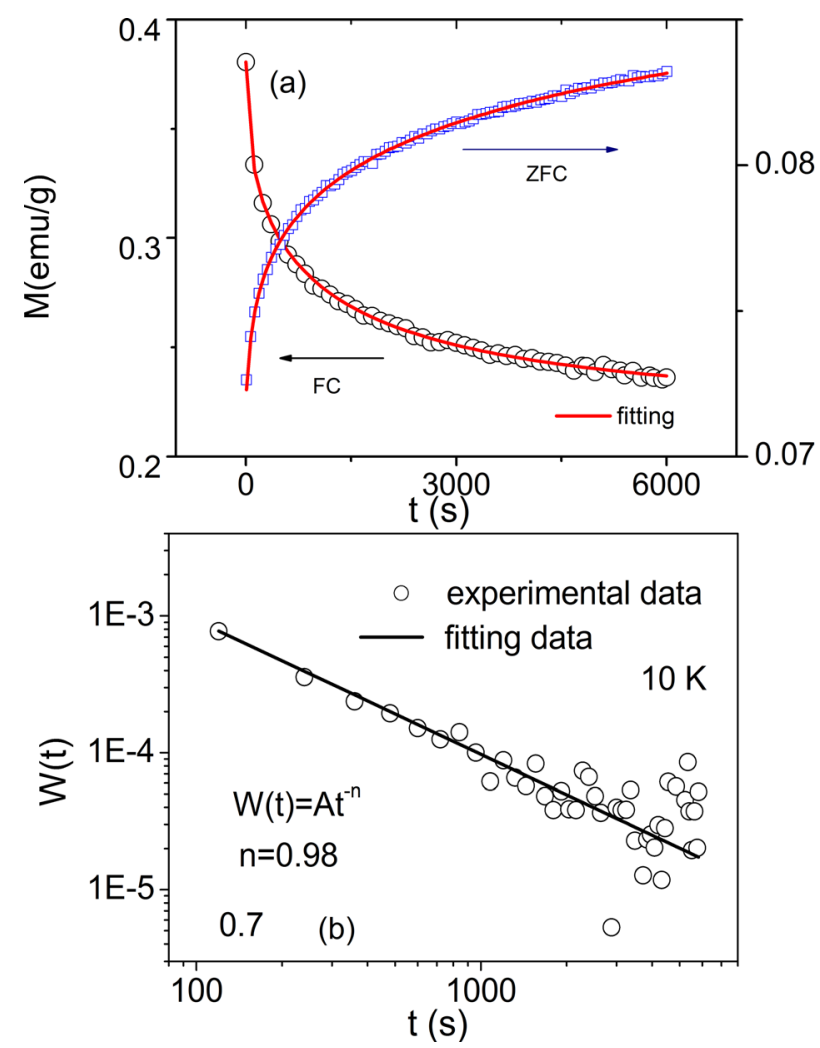

FIG. 4. (Color online) (a) Magnetic relaxation phenomena of ZFC and FC processes at $10 \mathrm{~K}$. Solid line show fitted data. (b) the relaxation rate of FC process.

zero fields. After waiting $600 \mathrm{~s}$, a dc magnetic field $(H=1$ kOe) was applied and the magnetization $M$ was recorded versus time $t$, as shown in Fig. 4(a). Obviously, the magnetic moment increased with increasing measured time. This magnetic relaxation curves $M(t)$ in SG system can be described by the stretched exponential form $\mathrm{M}(\mathrm{t})=M_{0}-M r \exp \left[-(\mathrm{t} / \tau)^{1-\mathrm{n}}\right]{ }^{16}$ where $M_{0}\left(M_{0}\right.$ is the magnetization at $\left.t=0\right)$ and $\tau$ depend on temperature $T$ and waited time $t_{w}$, while $\mathrm{n}$ is only a function of temperature $T$. Figure 4(a) shows that the experimental data are well represented by this equation, and the fitted parameters values are $\tau=4.8 \times 10^{3} \mathrm{~s}$ and $n=0.65$, respectively. We also estimated the aging effect for SMFMO, with the following procedure. The sample was cooled from 300 to $10 \mathrm{~K}$ in a magnetic field of $1 \mathrm{kOe}$. After waiting $600 \mathrm{~s}$, the magnetic field was switched off and the magnetization $M$ was recorded versus time $t$. The magnetic moment decreases with increasing time, and the FC $M(t)$ curve can be described by $M(t)=M_{0} \exp \left[-(t / \tau)^{1-n}\right]$, as shown in Fig. 4(a), where $M_{0}\left(M_{0}\right.$ is the magnetization at $\left.t=0\right)$ and $\tau$ depend on temperature $T$ and waiting time $t_{w}$, while $\mathrm{n}$ is only a function of temperature $T$. The fitted parameter values are $\tau=1.0 \times 10^{3} \mathrm{~s}$ and $n=0.47$, respectively. These fitted results confirm that the SMFMO exhibits spin glass behavior, which is consistent with the ac susceptibility. The relaxation rate in interacting magnetic particles of FC process, $W(t)=-(d / d t) \ln M(t)$, decays by a power law $W(t)=A t^{-n}$ (Ref. 17). The decay behavior of $W(t)$ can be well described by this equation and $n=0.98$, as shown Fig. 4(b). These results of relaxation in FC process suggest that the interaction between the ferromagnetic and antiferromagnetic clusters makes contribution to the spin glass behavior in SMFMO.

\section{CONCLUSIONS}

In summary, we have presented the magnetic data of $\mathrm{Sr}_{2} \mathrm{Mn}_{0.7} \mathrm{Fe}_{0.3} \mathrm{MoO}_{6}$ in detail. The dc magnetization, ac susceptibility, aging experiments and fitting results show spin glass behavior in the sample. The spin glass behavior originates from the competition between the ferromagnetic and antiferromagnetic couplings due to the magnetic frustration at the interface of ferromagnetic and antiferromagnetic clusters in the phase separate sample.

\section{ACKNOWLEDGMENTS}

This work was supported by the National Natural Science Foundation of China (10804024), China Postdoctoral Science Foundation funded project (20090450119 and 201003422), the Natural Scientific Research Innovation Foundation in Harbin Institute of Technology (HIT.NSRIF. 2008.03), the Program for New Century Excellent Talents in University (NCET-06-335), NSF (DMR-0852862).

${ }^{1}$ K.-I. Kobayashi, T. Kimura, H. Sawada, K. Terakura, and Y. Tokura, Nature (London) 395, 677 (1998).

${ }^{2}$ R. I. Dass, J.-Q. Yan, and J. B. Goodenough, Phys. Rev. B 68, 064415 (2003).

${ }^{3}$ X. J. Wang, Y. Sui, Y. Li, L. Li, X. Q. Zhang, Y. Wang, Z. G. Liu, W. H. Su, and J. K. Tang, Appl. Phys. Lett. 95, 252502 (2009).

${ }^{4}$ X. M. Feng, G. H. Rao, G. Y. Liu, H. F. Yang, W. F. Liu, Z. W. Ouyang, L. T. Yang, Z. X. Liu, R. C. Yu, C. Q. Jin, and J. K. Liang, J. Phys.: Condens. Matter 14, 12503 (2002).

${ }^{5}$ Z. Yang and L. Ye, J. Phys.: Condens. Matter 17, 2035 (2005).

${ }^{6}$ Y. Moritomo, H. Kusuya, A. Machida, E. Nishibori, M. Takata, M. Sakata, and A. Nakamura, J. Phys. Soc. Jpn. 70, 3182 (2001).

${ }^{7}$ E. N. Caspi, J. D. Jorgensen, M. V. Lobanov, and M. Greenblatt, Phys. Rev. B 67, 134431 (2003).

${ }^{8}$ A. Poddar and C. Mazumdar, J. Appl. Phys. 106, 093908 (2009).

${ }^{9}$ A. Poddar, R. N. Bhowmik, I. P. Muthuselvam, and N. Das, J. Appl. Phys. 106, 073908 (2009).

${ }^{10}$ H. M. Rietveld, Acta Crystallogr. 22, 151 (1967).

${ }^{11}$ J. Rodríguez-Carvajal, Physica B 192, 55 (1993).

${ }^{12}$ D. Rubi, C. Frontera, J. Nogués, and J. Fontcuberta, J. Phys.: Condens. Matter 16, 3173 (2004).

${ }^{13}$ Y. Sui, X. J. Wang, Z. N. Qian, J. G. Cheng, Z. G. Liu, J. P. Miao, Y. Li, W. H. Su, and C. K. Ong, Appl. Phys. Lett. 85, 269 (2004).

${ }^{14}$ Ll. Balcells, J. Navarro, M. Bibes, A. Roig, B. Martínez, and J. Fontcuberta, Appl. Phys. Lett. 78, 781 (2001).

${ }^{15}$ D. N. H. Nam, R. Mathieu, P. Nordblod, N. V. Khiem, and N. X. Phuc, Phys. Rev. B 62, 8989 (2000).

${ }^{16}$ G. C. DeFotis, G. S. Coker, J. W. Jones, C. S. Branch, H. A. King, J. S. Bergman, S. Lee, and J. R. Goodey, Phys. Rev. B 58, 12184 (1998).

${ }^{17}$ M. Ulrich, J. Garcia-Otero, J. Rivas, and A. Bunde, Phys. Rev. B 67, 024416 (2003). 